\title{
A domain containing the ESR motif in ENHANCER OF SHOOT REGENERATION 1 functions as a transactivation domain
}

\author{
Yoshihiro Nomura ${ }^{1, \dagger}$, Naoki Matsuo ${ }^{2, \dagger}$, Hiroharu Banno ${ }^{1, *}$ \\ ${ }^{1}$ Department of Environmental Biology, College of Bioscience and Biotechnology, Chubu University, Kasugai, Aichi \\ 487-8501, Japan; ${ }^{2}$ Plant Biology Research Center, Chubu University, Kasugai, Aichi 487-8501, Japan \\ *E-mail: hirobanno@isc.chubu.ac.jp Tel: +81-568-51-6242 Fax: +81-568-52-6594
}

Received May 18, 2009; accepted July 10, 2009 (Edited by T. Mizoguchi)

\begin{abstract}
The Arabidopsis ENHANCER OF SHOOT REGENERATION 1 (ESR1) gene is thought to regulate the initiation of in vitro shoot regeneration. ESRI encodes a transcriptional activator belonging to the ETHYLENE-RESPONSIVE FACTOR family. In this study, we investigated the functions of the C-terminal domain of the ESR1 protein. Effectorreporter assays using the GAL4 DNA-binding domain fused with portions of the C-terminal domain revealed that the ESR motif, which is a short amino acid sequence commonly seen in the C-terminal domain of ESR1 and ESR2, functions as a transactivation domain. We also examined the effects of partial deletions of the C-terminal domain on the in vitro shoot regeneration-enhancing effects of ESR1 overexpression. The results revealed that the ESR motif and a region between the AP2/ERF domain and the ESR motif were required for the shoot regeneration-enhancing effects of ESR1. The region between the AP2/ERF domain and the ESR motif appeared to have an essential function other than nuclear localization, DNA-binding, and transactivation. Substitution of the ESR motif by the VP16 transactivation domain abolished the ability of ESR1 to enhance shoot regeneration, suggesting that the ESR motif not only has transactivation activity but also has other functions.
\end{abstract}

Key words: $\quad E S R 1, E S R 2$, shoot regeneration, transcription factor.

Shoot regeneration is a key step for the successful production of transgenic plants in many species. Therefore, the elucidation of mechanisms regulating shoot differentiation in tissue culture is important for molecular breeding and biotechnology. The Arabidopsis ENHANCER OF SHOOT REGENERATION 1 (ESRI) [also known as DORNRÖSCHEN; DRN (Kirch et al. 2003)] is thought to regulate commitment to shoot differentiation, and ESR1 overexpression greatly enhances the efficiency of shoot regeneration in Arabidopsis tissue culture (Banno et al. 2001). ESR1 encodes a transcription factor belonging to the ETHYLENE-RESPONSIVE FACTOR (ERF) family. The ERF family is the largest branch in the AP2/ERF superfamily of transcription factors containing 1 or 2 AP2/ERF domains, which are plant-specific DNAbinding motifs (McGrath et al. 2005; Nakano et al. 2006; Shigyo et al. 2006). The Arabidopsis genome has more than 100 ERF family genes, which are implicated in diverse physiological events such as hormone signaling (Guo and Ecker 2004; Ohme-Takagi and Shinshi 1995; Song et al. 2005; van der Fits and Memelink 2000), biotic and abiotic stress tolerance (Agarwal et al. 2006;
Gutterson and Reuber 2004), cuticular wax biosynthesis (Aharoni et al. 2004; Broun et al. 2004; Zhang et al. 2005), and developmental processes (Banno et al. 2001; Chandler et al. 2007; Hirota et al. 2007; Ikeda et al. 2006; Kirch et al. 2003; van der Graaff et al. 2000). Some members of the ERF family are known to function as transactivators and others, as transcriptional repressors. We previously demonstrated that ESR1 acts as a transcriptional activator (Matsuo and Banno 2008). The Arabidopsis genome has another gene, ESR2 (Ikeda et al. 2006) [also named $D R N L$ (Kirch et al. 2003), SOB2 (Ward et al. 2006), and BOLITA (Marsch-Martinez et al. 2006)]. ESR2 overexpression also enhanced in vitro shoot regeneration (Ikeda et al. 2006), implying that ESR1 and ESR2 function redundantly in the regulation of shoot regeneration.

Here, we used the DNA-binding domain of the yeast GAL4 transcription factor (GAL4DBD) (Keegan et al. 1986) fused to partial regions of ESR1 to locate a transactivating domain in a short peptide commonly seen in the C-terminal regions of ESR1 and ESR2. We also examined the effects of the overexpression of ESR1 with partial deletions of the $\mathrm{C}$-terminal region on in vitro

\footnotetext{
${ }^{\dagger}$ These authors contributed equally to this work.

Abbreviations: CIM, callus-inducing medium; 2,4-D, 2,4-dichlorophenoxyacetic acid; 2-ip, $\mathrm{N}^{6}-\Delta^{2}$-isopentenyladenine; MS, Murashige and Skoog; PCR, polymerase chain reaction; SIM, shoot-inducing medium.

This article can be found at http://www.jspcmb.jp/
} 
shoot regeneration.

\section{Material and methods}

\section{Plant materials and media}

Arabidopsis thaliana Wassilewskija (WS) plants were used for the root cultures and $A$. thaliana Columbia (Col-0) plants were used as targets for particle bombardment. The MS medium contained MS salts (Wako Pure Chemical Industries Ltd.), 0.1\% Gamborg's B5 vitamins (Sigma-Aldrich Co.), 1\% sucrose, and $0.3 \%$ gellan gum (Wako Pure Chemical Industries Ltd.). The CIM contained Gamborg's B5 salts (Sigma-Aldrich Co.), $2 \%$ glucose, $0.1 \%$ Gamborg's B5 vitamins, $2 \mu \mathrm{M}$ 2,4-D, $40 \mu \mathrm{M}$ acetosyringone, and $0.6 \%$ gellan gum. The SIM contained MS salts, $1 \%$ sucrose, $0.1 \%$ Gamborg's B5 vitamins, $12.5 \mu \mathrm{M} 2$-ip, and $0.3 \%$ gellan gum. The $\mathrm{C}$ medium comprised SIM supplemented with $0.4 \mathrm{gl}^{-1}$ carbenicillin and $50 \mathrm{mgl}^{-1}$ kanamycin.

\section{Construction of plasmids}

The construction of pTALuc, p35S:: GAL4DBD, p35S:: GAL4DBD-VP16, and p35S::GAL4DBD-ESR1C has been described previously (Matsuo and Banno 2008). Fragments encoding ESR1C $\Delta 1$ ESR1C $\Delta 2$ and ESR1C $\Delta 3$ (C-terminal domains of ESR1 with partial deletions) were amplified by PCR from p35S::GAL4DBD-ESR1C using the following primers: vector primer ( $5^{\prime}$-CTCTATATAAGGAAGTTCATTTCATTTGG-3') and ESR1C-del 1 (5'-AGCGGCCGCACTAGTCTAGTCGCGTAAGAAGATAGACTGTGTA-3') for ESR1C $\Delta 1$; vector primer and ESR1C-del 2 (5'-AGCGGCCGCACTAGTCTATTCCGGGAAAAACGACTCGTT-3') for ESR1C $\Delta 2$; and vector primer and ESR1C-del 3 (5'AGCGGCCGCACTAGTCTATGTTGGTGGAGAAGGAGGA $\left.\mathrm{AC}-3^{\prime}\right)$ for ESR1C $\Delta 3$. The amplified fragments were inserted between the $A s c \mathrm{I}$ and NotI sites in pSK34 after digestion with $A s c \mathrm{I}$ and NotI, resulting in p35S:: GAL4DBD-ESR1C $\Delta 1$, p35S::GAL4DBD-ESR1C $\Delta 2$, and p35S :: GAL4DBDESR1C $\Delta 3$. p35S :: GAL4DBD-EM1 and p35S :: GAL4DBDEM2 were constructed by replacing the C-terminal domain of ESR1 in p35S :: GAL4DBD-ESR1C by a region containing the ESR motif (EM1 or EM2). The fragments encoding GAL4DBD were excised from p35S :: GAL4DBD-ESR1C. The fragments encoding the ESR motif were amplified from pSKESR1 (Banno et al. 2001) by PCR using the primers C1F (5'GAGAGAGAATTCGAAAACAACGAGTCGTTTTTCCC-3') and C1R2 (5'-CTCGAGACTAGTTGTCATGATGGCTAGTCACCG-3') for EM1 or C1F2 (5'-ACCTAGGCCGGAAGAATCTTCTGATACTG-3') and ESR1C-del 3 for EM2. Then, the fragments encoding GAL4DBD and those encoding the Cterminal region of ESR1 containing the ESR motif were coinserted between the AscI and NotI sites in pSK34 to generate p35S :: GAL4DBD-EM1 or p35S :: GAL4DBD-EM2. pBI221 (p35S:: GUS; Clontech Laboratories Inc.) was used as a reference plasmid.

The construction of pER10::ESR1 has been described previously (Banno et al. 2001). Fragments encoding ESR $1 \Delta 2$ and those encoding ESR $1 \Delta 3$ were amplified from pSK-ESR1 by PCR using the vector primer and ESR1C-del 2 for ESR1 $\Delta 2$ and the vector primer and ESR1C-del 3 for ESR1 $\Delta 3$. These fragments were inserted into pER10 (Zuo et al. 2000) between the AscI and SpeI sites after digestion with $A s c \mathrm{I}$ and SpeI to produce pER10::ESR1 $\Delta 2$ and pER10::ESR1 $\Delta 3$. The fragments encoding ESR1 $\Delta 2 /$ AvrII (ESR1 $\Delta 2$ with $A v r I I$ site at its C-terminal end) were amplified from pSK-ESR1 by PCR using the vector primer and ESR1C-del 2-AvrII (5'ACCTAGGTTCCGGGAAAAACGACTCGTT-3'). The fulllength sequence of ESR1 in pER10:: ESR1-VP16 (Matsuo and Banno 2008) was replaced by ESR $1 \Delta 2 / A v r I I$ to generate pER10:: ESR1 $\Delta 2$-VP16. The fragments encoding the Cterminal region containing the ESR motif were amplified from pSK-ESR1 by PCR using the primers C1F and C1R2. These fragments were used to replaced the N-terminal region of ESR1 in pER10 :: ESR1N-VP16 (Matsuo and Banno 2008), resulting in $\mathrm{pER} 10::$ ESR1N-EM1.

\section{Effector-reporter transient expression assays}

The constructed plasmids were transiently transformed and the luciferase activity was determined as described previously (Matsuo and Banno 2008).

\section{Shoot regeneration assays}

Arabidopsis seeds were sterilized and sown on MS medium and grown for 2 weeks at $22^{\circ} \mathrm{C}$ under continuous lighting. Roots were then cut into approximately $1-\mathrm{cm}$ segments and used for tissue culture. Root segments preincubated on CIM for 3 days were co-cultured for 3 days with Agrobacterium tumefaciens EHA105 carrying an appropriate plasmid; the root segments were washed three times with sterilized water and then incubated on $\mathrm{C}$ medium with or without $5 \mu \mathrm{M} 17 \beta$-estradiol. The number of shoots generated in the root cultures was determined after 4 weeks.

\section{Results}

\section{Transactivation by the ESR motif}

ESR2 encodes a protein that is structurally related to ESR1 (Ikeda et al. 2006), and ESR2 overexpression also enhances in vitro shoot regeneration as is the case with ESR1, implying that ESR1 and ESR2 function redundantly in the regulation of shoot regeneration (Mase et al. 2007). Figure 1A represents the schematic structure of the ESR1 and ESR2 proteins, and Figure 1B shows the alignment of amino acid sequences of their AP2/ERF domains. The amino acid sequences in the AP2/ERF domains of ESR1 and ESR2 are very similar (93\% identical); however, except for a short amino acid sequence (designated as the ESR motif) in the Cterminal domain of ESR1 and ESR2, the other domains do not display significant structural similarity (Figure 1C). Although ESL3, another putative protein in Arabidopsis, also has an ESR motif-like sequence, the similarity in the amino acid sequence of the short homologous stretch is considerably low between ESL3 and the other proteins as compared to that between ESR1 and ESR2. Further, the role of ESL3 in the regulation of shoot regeneration is not redundant unlike the roles of ESR1 and ESR2 (Mase et al. 2007). We have previously 
A

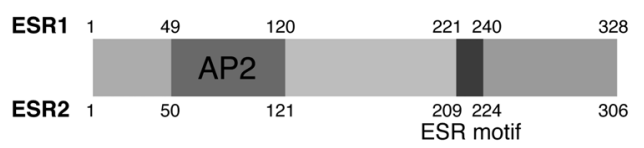

B

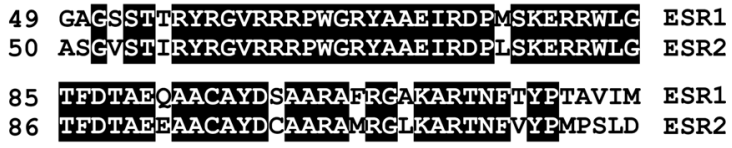

C

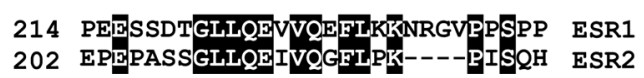

Figure 1. Structural characteristics of ESR1 and ESR2. (A) Schematic representation of the ESR1 and ESR2 proteins. "AP2" indicates the AP2/ERF domain. Amino acid numbers are indicated above (ESR1) and below (ESR2) the figure. (B) Alignment of the amino acid sequences of the AP2/ERF domains of ESR1 and ESR2. Black shading indicates identical amino acid residues. Amino acid numbers are indicated on the left. (C) Alignment of the amino acid sequences of the ESR motifs of ESR1 and ESR2. Black shading indicates identical amino acid residues. Amino acid numbers are indicated on the left.

reported that ESR1 acts as a transcriptional activator and its transactivation domain is located in the C-terminal region after the AP2/ERF domain (Matsuo and Banno 2008). In order to define the transactivation domain to a smaller region in the $\mathrm{C}$-terminal domain, reportereffector assays were carried out. A deletion series of the C-terminal domain was fused to GAL4DBD, as shown in Figure 2A. The transactivation domain of the herpes viral protein VP16 (Triezenberg et al. 1988) was also fused to GAL4DBD (GAL4DBD-VP16) to serve as a positive control. The coding region of the luciferase gene (LUC) under the control of the 6xUAS promoter [consisting of 6 copies of the GAL4 upstream activating sequence (Giniger et al. 1985) and the 35S minimal promoter] was used as the reporter gene in the transient expression assays. Each effector plasmid, the reporter plasmid, and the reference plasmid were delivered into Arabidopsis leaf cells by particle bombardment and the luciferase activity was determined (Figure 2B). GAL4DBD-VP16 overexpression clearly elevated the luciferase activity. When GAL4DBD-ESR1C was overexpressed, luciferase activity increased approximately 5.5 -fold as compared to the activity observed with GAL4DBD alone. While the overexpression of ESR1C $\Delta 1$ and ESR1C $\Delta 2$ fused to GAL4DBD did not significantly increase the luciferase activity, ESR1C $\Delta 3$ overexpression increased the luciferase activity 3.9-fold as compared to the activity obtained with GAL4DBD alone. Expression of GAL4DBD alone enhanced the basal luciferase activities to a greater extent than the basal green fluorescent protein (GFP) activities. Expression of GAL4DBDESR1C $\Delta 1$ or GAL4DBD-ESR1C $\Delta 2$ also induced basal luciferase activities; this result suggests that these proteins are expressed in plant cells. Together, these findings suggested that the C-terminal domain of ESR1 requires the ESR motif for transactivation. We also examined whether the ESR motif alone was able to activate the transcription of the reporter gene. For this purpose, a C-terminal region (amino acids 207-252) containing the ESR motif was fused to GAL4DBD and the resulting construct (GAL4DBD-EM1) was subjected to the effector-reporter assay. The expression of GAL4DBD-EM1 increased the luciferase activity (5.3fold) to a level comparable to that observed with the fulllength $\mathrm{C}$-terminal domain. A smaller region (amino acids 214-243) was also fused to the GAL4DBD (GAL4DBDEM2). GAL4DBD-EM2 increased the luciferase activity (2.7-fold) to a lesser extent than GAL4DBD-EM1, but still retained the ability to transactivate the reporter gene. These results clearly demonstrated that the ESR motif has the ability to activate transcription.

\section{Effects of overexpression of ESR1 with partial deletions in the C-terminal domain on shoot regeneration}

The above results indicate that the ESR motif functions as a transactivation domain. In order to examine whether the ESR motif is required for enhancing shoot regeneration, a deletion series of ESR1 was overexpressed during in vitro shoot regeneration. ESR1 cDNAs with a series of partial deletions in the Cterminal region, as shown in Figure 3, were inserted into the estrogen-inducible expression vector pER10. The constructs were transformed into root explants cut from Arabidopsis plants and were preincubated on CIM for 3 days. After incubation for 4 weeks on $\mathrm{C}$ medium with or without $17 \beta$-estradiol, the regenerated shoots were counted (Figure 3). The induction of ESR1 overexpression by estrogen enhanced shoot regeneration (11.3-fold) when compared to that without estrogen (-ED). The overexpression of ESR1 with a deletion after the ESR motif $(\operatorname{ESR} 1 \Delta 3)$ continued to have enhancing effects (9.4-fold) on shoot regeneration, although the effects decreased to approximately $85 \%$ of those elicited by full-length ESR1. In contrast, the shoot regeneration-enhancing effects were abolished when a Cterminal region containing the ESR motif was deleted (ESR1 $\Delta 2)$. These results indicate that the ESR motif is indispensable for the shoot regeneration-enhancing effects of ESR1.

A region between the AP2/ERF domain and the ESR motif of ESR1 is required for its shoot regeneration-enhancing effects

We previously demonstrated that the AP2/ERF domain preferentially bound to the sequence A/TGCCGCC (Banno et al. 2006) and a nuclear localization sequence was also located in the AP2/ERF domain (Matsuo and 


\section{A Reporter plasmid}

pTALUC 6XGAL4 UAS TATA LUC

Effector plasmid

p35S::GFP 35S promoter GFP

p35S::GAL4DBD 35S promoter GAL4DBD

p35S::GAL4DBD-ESR1C 35S promoter GAL4DBD ${ }^{123}$ ESR motif

p35S::GAL4DBD-ESR1C $\Delta 1$ 35S promoter GAL4DBD

p35S::GAL4DBD-ESR1C $\triangle 2$ 35S promoter GAL4DBD

p35S::GAL4DBD-ESR1C $\triangle 3$ 35S promoter GAL4DBD 240

p35S::GAL4DBD-EM1 35S promoter GAL4DBD

ESR mo
214243

p35S::GAL4DBD-EM2 35S promoter GAL4DBD

Reference plasmid

ESR motif

p35S::GUS 35S promoter GUS

B

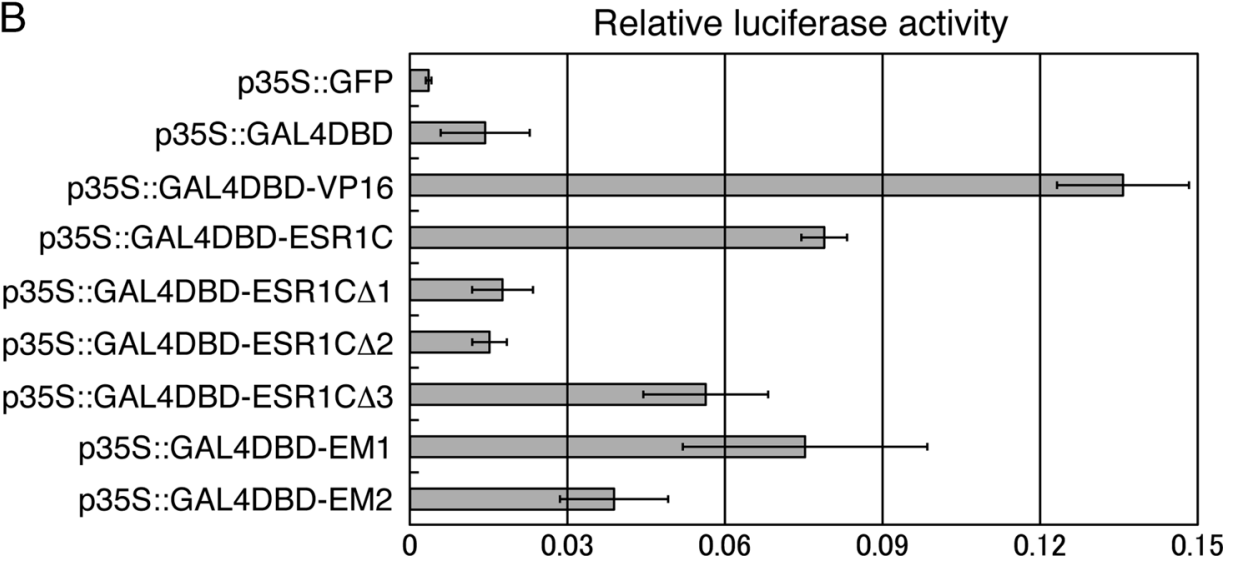

Figure 2. Transactivation of the reporter gene by the C-terminal regions of ESR1. (A) Schematic representations of the reporter, effector, and reference plasmids used in the transient expression assays. Amino acid numbers corresponding to the wild-type ESR1 protein are indicated above the schematic representations of the fusion proteins. Effector and reference cDNAs were expressed under the control of the 35S promoter. (B) Relative luciferase activity induced by the overexpression of the effector cDNAs. LUC activity was normalized against GUS activity. The data represent the average of 3 samples, and error bars indicate standard deviations.

Banno 2008), whereas the N-terminal region before the AP2/ERF domain was not required for the enhancing effects of ESR1 on in vitro shoot regeneration. Generally, a DNA-binding domain(s), transcription-modulating domain(s), and nuclear localization signal(s) are required for the activities of transcription factors (Liu et al. 1999). We examined whether a combination of the AP2/ERF domain and the ESR motif was sufficient for ESR1 activity, using the shoot regeneration assay summarized in Figure 3. The N-terminal region (amino acids 1-122) containing the AP2/ERF domain was fused with the Cterminal region (amino acids 207-252) containing the ERF motif (ESR1N-EM1) and inserted into the estrogeninducible vector pER10. However, ESR1N-EM1 overexpression did not enhance shoot regeneration significantly (Figure 4). These results suggest that a 

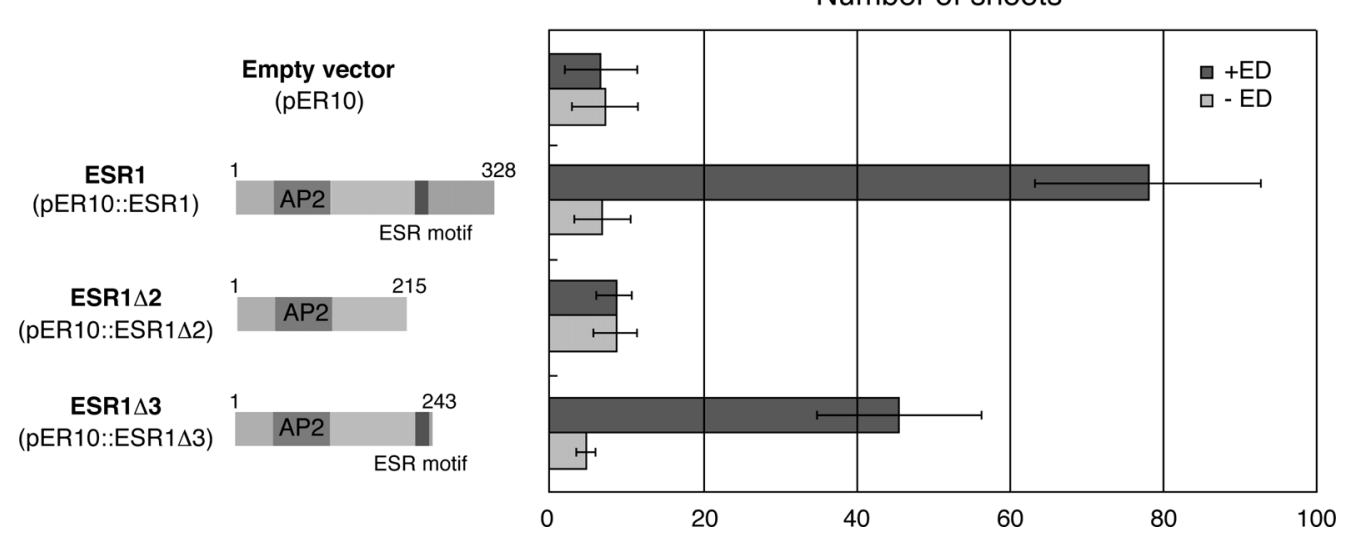

Figure 3. Effects of the overexpression of ESR1 with partial deletions in the C-terminal region on shoot regeneration. The number of transformed shoots obtained from $0.1 \mathrm{~g}$ (fresh weight) of root culture. Arabidopsis root cultures preincubated on CIM for 3 days were transformed with the constructs indicated on the left and were then incubated on the $\mathrm{C}$ medium with $(+\mathrm{ED})$ or without $(-\mathrm{ED})$ a transcription inducer $(5 \mu \mathrm{M} 17 \beta$ estradiol) for 4 weeks. The data represent the average of 3 experiments, and error bars indicate standard deviations. "AP2" indicates the AP2/ERF domain. Amino acid numbers corresponding to the wild-type ESR1 protein are indicated above ESR1 and its derivative proteins.

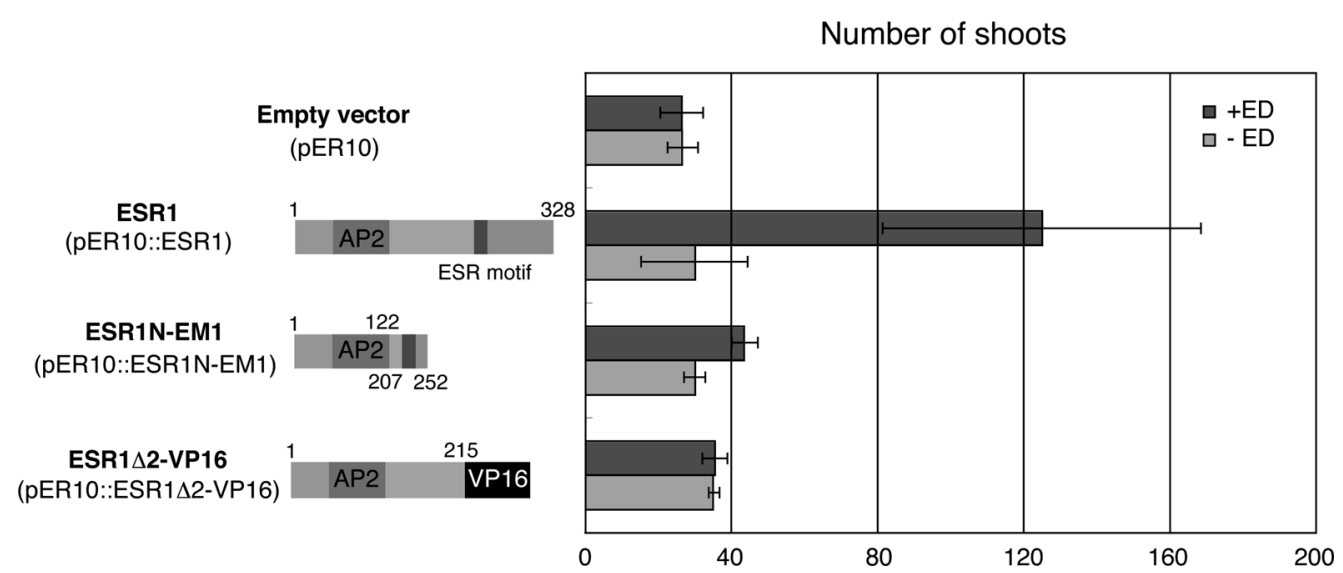

Figure 4. Effects of the overexpression of ESR1 with partial deletions in the C-terminal region or with substitution of the transactivation domain on shoot regeneration. The number of transformed shoots obtained from $0.1 \mathrm{~g}$ (fresh weight) of root culture. Arabidopsis root cultures preincubated on CIM for 3 days were transformed with the constructs indicated on the left, and were then cultured on the $\mathrm{C}$ medium with (+ED) or without ( $-\mathrm{ED}) \mathrm{a}$ transcription inducer ( $5 \mu \mathrm{M} 17 \beta$-estradiol) for 4 weeks. "AP2" indicates the AP2/ERF domain. Amino acid numbers corresponding to the wild-type ESR1 protein are indicated above or below the schematic representations of ESR1 and its derivative proteins. The data represent the average of 3 experiments, and the error bars indicate standard deviations.

combination of the AP2/ERF domain and the ESR motif is not sufficient for ESR1 activity, and a region between the AP2/ERF domain and the ESR motif is also required for the shoot regeneration-enhancing effects of ESR1. We also examined whether it is possible to substitute the ESR motif by a transactivating peptide, VP16. The ESR motif of ESR1 $\Delta 3$ (Figure 3) was exchanged with VP16 (ESR1 $\Delta 2$-VP16) and was inserted into pER10. However, ESR1 $\Delta 2$-VP16 overexpression did not have shoot regeneration-enhancing effects (Figure 4). These results suggest that the ESR motif not only has the ability of transcriptional activation but also has other indispensable functions.

\section{Discussion}

We previously reported that ESR1 acts as a transcriptional activator and its transactivation domain is located in the $\mathrm{C}$-terminal region after the AP2/ERF domain (Matsuo and Banno 2008). The results of effector-reporter assays have revealed that the C-terminal region of ESR1 lost the ability of transactivation on deletion of the ESR motif and that a small region containing the ESR motif exhibited the transactivation ability when fused to GAL4DBD (Figure 2). These results clearly demonstrated that transcriptional activation of ESR1 was attributed to the small region containing the ESR motif. The ESR motifs of both ESR1 and ESR2 also exhibit transactivation ability in yeast cells (our unpublished results), although we have not 
examined transactivation by the ESR motifs of ESR2 in plant cells. We also demonstrated that the ESR motif was indispensable for the shoot regeneration-enhancing effects of ESR1 (Figure 3). The overexpression of ESR1N-EM1 (containing the AP2/ERF domain with DNA-binding ability and a nuclear localization signal and the ESR motif with transactivation ability) did not enhance shoot regeneration (Figure 4), suggesting that a region between the AP2/ERF domain and the ERF motif is also required for ESR1 activity. In addition, substitution of the ESR motif by VP16 abolished the shoot regeneration-enhancing effects of ESR1 (Figure 4). The ESR motif appeared to have not only transactivation ability but also other functions. The deletion of a region after the ESR motif slightly decreased both the transactivation of the reporter gene (Figure 2) and shoot regeneration (Figure 3 ). The deletion of this region may have affected the stability of the ESR1 protein.

The optimal binding sequence of the AP2/ERF domain of ESR1 is the GCC box (the sequence AGCCGCC), which is known to be an ethyleneresponsive element (Banno et al. 2006). Many pathogenesis-related genes have ethylene-responsive elements in their promoters (Guo and Ecker 2004; Ohme-Takagi and Shinshi 1995). Is it possible that the GCC box alone differentially regulates defense response and shoot regeneration? It has been reported that flanking sequences can affect the binding of ERFs to the GCC box (Gu et al. 2002; Tournier et al. 2003); however, no consensus sequence was observed outside the $\mathrm{A} / \mathrm{TGCCGCC}$ sequence in the ESR1 binding sequences identified by binding sequence selection assay (our unpublished results). Alternatively, ESR1 may preferentially bind to sequences other than the GCC box in vivo owing to the modulation of binding specificity by another factor. Recently, it has been reported that the AP2/ERF domain of DRN/ESR1 interacts with class III HD-ZIP transcription factors (Chandler et al. 2007) and BIM1, which is a helix-loop-helix transcription factor (Chandler et al. 2009). Interaction of these transcription factors with the AP2/ERF domain of DRN/ESR1 may alter the binding specificity of DRN/ESR1. Another possibility is that a complex of ESR1 and another transcription factor may recognize an extended sequence containing a GCC box. A region between the AP2/ERF domain and the ESR motif was required for ESR1 activity. The ESR motif also has essential functions other than transactivation. These regions in the $\mathrm{C}$-terminal domain of ESR1 may interact with other factors and regulate downstream genes through a combination of their recognition sequences, although such factors still need to be identified. At present we are attempting to identify the proteins interacting with the C-terminal domain of ESR1 by yeast two-hybrid screening. Such a study may lead to the elucidation of the differential regulation of defense response and shoot regeneration by the ERF family.

\section{Acknowledgements}

This research was supported by the Academic Frontier Project for Private Universities matching fund subsidy from Ministry of Education, Culture, Sports, Science and Technology (MEXT) of Japan, 2005-2009.

\section{References}

Agarwal PK, Agarwal P, Reddy MK, Sopory SK (2006) Role of DREB transcription factors in abiotic and biotic stress tolerance in plants. Plant Cell Rep 25: 1263-1274

Aharoni A, Dixit S, Jetter R, Thoenes E, van Arkel G et al. (2004) The SHINE clade of AP2 domain transcription factors activates wax biosynthesis, alters cuticle properties, and confers drought tolerance when overexpressed in Arabidopsis. Plant Cell 16: 2463-2480

Banno H, Ikeda Y, Niu QW, Chua NH (2001) Overexpression of Arabidopsis ESR1 induces initiation of shoot regeneration. Plant Cell 13: 2609-2618

Banno H, Mase H, Maekawa K (2006) Analysis of functional domains and binding sequences of Arabidopsis transcription factor ESR1. Plant Biotechnol 23: 303-308

Broun P, Poindexter P, Osborne E, Jiang CZ, Riechmann JL (2004) WIN1, a transcriptional activator of epidermal wax accumulation in Arabidopsis. Proc Natl Acad Sci USA 101: 4706-4711

Chandler JW, Cole M, Flier A, Grewe B, Werr W (2007) The AP2 transcription factors DORNROSCHEN and DORNROSCHENLIKE redundantly control Arabidopsis embryo patterning via interaction with PHAVOLUTA. Development 134: 1653-1662

Chandler JW, Cole M, Flier A, Werr W (2009) BIM1, a bHLH protein involved in brassinosteroid signalling, controls Arabidopsis embryonic patterning via interaction with DORNROSCHEN and DORNROSCHEN-LIKE. Plant Mol Biol 69: 57-68

Giniger E, Varnum SM, Ptashne M (1985) Specific DNA binding of GAL4, a positive regulatory protein of yeast. Cell 40: 767-774

Gu YQ, Wildermuth MC, Chakravarthy S, Loh YT, Yang C et al. (2002) Tomato transcription factors Pti4, Pti5, and Pti6 activate defense responses when expressed in Arabidopsis. Plant Cell 14: $817-831$

Guo H, Ecker JR (2004) The ethylene signaling pathway: new insights. Curr Opin Plant Biol 7: 40-49

Gutterson N, Reuber TL (2004) Regulation of disease resistance pathways by AP2/ERF transcription factors. Curr Opin Plant Biol 7: 465-471

Hirota A, Kato T, Fukaki H, Aida M, Tasaka M (2007) The auxinregulated AP2/EREBP gene PUCHI is required for morphogenesis in the early lateral root primordium of Arabidopsis. Plant Cell 19: 2156-2168

Ikeda Y, Banno H, Niu QW, Howell SH, Chua NH (2006) The ENHANCER OF SHOOT REGENERATION 2 gene in Arabidopsis regulates CUP-SHAPED COTYLEDON 1 at the transcriptional level and controls cotyledon development. Plant Cell Physiol 47: 1443-1456

Keegan L, Gill G, Ptashne M (1986) Separation of DNA binding 
from the transcription-activating function of a eukaryotic regulatory protein. Science 231: 699-704

Kirch T, Simon R, Grunewald M, Werr W (2003) The DORNROSCHEN/ENHANCER OF SHOOT REGENERATION 1 gene of Arabidopsis acts in the control of meristem ccll fate and lateral organ development. Plant Cell 15: 694-705

Liu L, White MJ, MacRae TH (1999) Transcription factors and their genes in higher plants functional domains, evolution and regulation. Eur J Biochem 262: 247-257

Marsch-Martinez N, Greco R, Becker JD, Dixit S, Bergervoet JH et al. (2006) BOLITA, an Arabidopsis AP2/ERF-like transcription factor that affects cell expansion and proliferation/differentiation pathways. Plant Mol Biol 62: 825-843

Mase H, Hashiba M, Mastsuo N, Banno H (2007) Expression patterns of Arabidopsis ERF VIII-b subgroup genes during in vitro shoot regeneration and effects of their overexpression on shoot regeneration efficiency. Plant Biotechnol 24: 481-486

Matsuo N, Banno H (2008) The Arabidopsis transcription factor ESR1 induces in vitro shoot regeneration through transcriptional activation. Plant Physiol Biochem 46: 1045-1050

McGrath KC, Dombrecht B, Manners JM, Schenk PM, Edgar CI et al. (2005) Repressor- and activator-type ethylene response factors functioning in jasmonate signaling and disease resistance identified via a genome-wide screen of Arabidopsis transcription factor gene expression. Plant Physiol 139: 949-959

Nakano T, Suzuki K, Fujimura T, Shinshi H (2006) Genome-wide analysis of the ERF gene family in Arabidopsis and rice. Plant Physiol 140: 411-432

Ohme-Takagi M, Shinshi H (1995) Ethylene-inducible DNA binding proteins that interact with an ethylene-responsive element. Plant Cell 7: 173-182

Shigyo M, Hasebe M, Ito M (2006) Molecular evolution of the AP2 subfamily. Gene 366: 256-265
Song CP, Agarwal M, Ohta M, Guo Y, Halfter U et al. (2005) Role of an Arabidopsis AP2/EREBP-type transcriptional repressor in abscisic acid and drought stress responses. Plant Cell 17: 2384-2396

Tournier B, Sanchez-Ballesta MT, Jones B, Pesquet E, Regad F et al. (2003) New members of the tomato ERF family show specific expression pattern and diverse DNA-binding capacity to the GCC box element. FEBS Lett 550: 149-154

Triezenberg SJ, Kingsbury RC, McKnight SL (1988) Functional dissection of VP16, the trans-activator of herpes simplex virus immediate early gene expression. Genes Dev 2: 718-729

van der Fits L, Memelink J (2000) ORCA3, a jasmonateresponsive transcriptional regulator of plant primary and secondary metabolism. Science 289: 295-297

van der Graaff E, Dulk-Ras AD, Hooykaas PJ, Keller B (2000) Activation tagging of the LEAFY PETIOLE gene affects leaf petiole development in Arabidopsis thaliana. Development 127: 4971-4980

Ward JM, Smith AM, Shah PK, Galanti SE, Yi H et al. (2006) A new role for the Arabidopsis AP2 transcription factor, LEAFY PETIOLE, in gibberellin-induced germination is revealed by the misexpression of a homologous gene, SOB2/DRN-LIKE. Plant Cell 18: 29-39

Zhang JY, Broeckling CD, Blancaflor EB, Sledge MK, Sumner LW et al. (2005) Overexpression of WXP1, a putative Medicago truncatula AP2 domain-containing transcription factor gene, increases cuticular wax accumulation and enhances drought tolerance in transgenic alfalfa (Medicago sativa). Plant $J 42$ : 689-707

Zuo J, Niu QW, Chua NH (2000) An estrogen receptor-based transactivator XVE mediates highly inducible gene expression in transgenic plants. Plant J 24: 265-273 